Unfallchirurg 2018 $121: 938-939$

https://doi.org/10.1007/s00113-018-0568-6

(C) Springer Medizin Verlag GmbH, ein Teil von Springer Nature 2018

CrossMark

\title{
P. Biberthaler
}

Klinik und Poliklinik für Unfallchirurgie, Klinikum rechts der Isar, Technische Universität München, München, Deutschland

\section{Versorgungsforschung}

Das Thema „Versorgungsforschung“ ist für die Unfallchirurgie bislang eher ein sehr entfernt wirkendes Thema. Dies ist sehr erstaunlich, da die Zukunft der Unfallchirurgie ganz wesentlich von den Ergebnissen der Versorgungsforschung abhängen wird. Bitte gestatten Sie, dass ich Ihnen die Hintergründe ein wenig erkläre:

Das Deutsche Netzwerk für Versorgungsforschung ist die wissenschaftliche Fachgesellschaft, die das Thema strukturiert und organisiert. In diesem $\mathrm{Zu}$ sammenhang wird es beispielsweise angefragt vom Institut für Qualitätssicherung und Transparenz im Gesundheitswesen (IQTIG), um Stellungnahmen zu wichtigen Fragestellungen, wie z. B. Qualitätsindikatoren zum Thema der Mindestmengenregelung oder ein Evaluationskonzept zu Qualitätsverträgen nach $\$ 110$ a SGB V, zu erstellen. Auf den ersten Blick mögen diese Begriffe etwas sperrig und sehr trocken wirken, aber bei näherer Betrachtung enthalten sie eine existenzielle Bedeutung für die Unfallchirurgie. So wurde die Endoprothetikals eine von 4 therapeutischen Komplexen identifiziert, für die ein Konzept durch das IQTIG im Auftrag des Gemeinsamen Bundesausschuss erarbeitet werden soll, um eine Einführung von Qualitätsverträgen planen zu können. Dabei ist nun völlig klar, dass der Messung der Qualität dabei größte Bedeutung zukommt.

Die Messung des Ergebnisses unfallchirurgischer Therapien war seit dem Beginn der Erfolgsgeschichte der Arbeitsgemeinschaft für Osteosynthesefragen (AO) ein zentraler Bestandteil der Entwicklung. Nur durch die konsequente Dokumentation und Auswertung der „neuen“ Operationstechniken war es möglich, die spektakulären innovativen Entwicklungen der AO voranzutreiben.
In der konsequenten Fortsetzung dieser Historie ist es daher unerlässlich, die Qualität der Ergebnisse der unfallchirurgischen Therapien möglichst umfassend zu messen. Da die Anzahl der Therapien und damit auch Operationen sich aber seit den ersten Tagen der AO aufgrund der spektakulären Erfolge so dramatisch nach oben entwickelt hat, ist eine Messung mit klassischen Instrumenten der arztbasierten Nachuntersuchung in der Breite derzeit nicht mehr möglich. Die neueren wissenschaftlichen Erkenntnisse über den Erfolg unfallchirurgischer Therapien beruhen derzeit im Wesentlichen auf entweder retrospektiven Kohortenoder kleinen, prospektiven randomisierten Studien (,randomized clinical trial“, RCT). Auch wenn klar ist, dass in anderen Bereichen der Medizin, wie z.B. der pharmakologischen Therapie die RCT das beste Mittel ist, um Therapien zu beurteilen, so sind für die operativen Fächer in der jüngeren Vergangenheit einige Fragen aufgetreten: Es dürfte jedem klar sein, dass man ein operatives Verfahren nur einseitig verblinden kann, und jeder Operateur hat ein Verfahren, welches er aufgrund von positiven Erfahrungen etc. bevorzugt. Um die operative Expertise auszugleichen, hat man versucht, Operateure in beiden Verfahren zu schulen, aber den langjährigen Erfahrungshintergrund wird man damit als entscheidenden „confounder“ damit nicht eliminieren können. Somit bilden RCT vielleicht nur eine „künstliche“ Realität ab, die mit der realen Versorgungsrealität nicht unbedingt etwas $\mathrm{zu}$ tun haben muss. Hieraus könnte sich auch die Diskrepanz erklären, zwischen der klinischen Erfahrung über bestimmte therapeutische Verfahren, die sich in RCT aber nicht immer nachweisen lässt. In diesem Spannungsfeld bietet nun 
das relativ junge Gebiet der Versorgungsforschung relevante und gute Lösungsmöglichkeiten an. Die konsequente Anwendung von neuesten Technologien insbesondere auf dem Gebiet der Datenverarbeitung hat gezeigt, dass sich mit der Erfassung von großen Datenmengen auch in einem retrospektiven Setting relevante Aussagen über die Qualität von Behandlungen erzielen lassen. Wenn man diese Erfassungstechnologien noch in den Bereich von patientenzentrierten Ergebnismessungen bringt, wird die Schlagkraft der Methoden von Versorgungsforschung deutlich: Nun steht dem Kliniker ein „Labor“ zur Verfügung, mit dem er unter Verwendung eines begrenzten Aufwandes hochrelevante Daten über die Ergebnisse seiner Behandlung generieren kann. Daher freue ich mich ganz besonders, dass Herr Prof. Neugebauer sich bereit erklärt hat, in seinem Artikel die Grundlagen der Versorgungsforschung und deren ganze Bandbreite der Techniken zu erläutern, die natürlich weit über die von mir beispielhaft skizzierten Registerstudien hinausgehen. Gerade in den skandinavischen Ländern haben uns die Kollegen eindrucksvoll gezeigt, welche überzeugenden Daten aus großen Registern generiert werden können. Daher freue ich mich ganz besonders, dass Herr Dr. Möller aus Göteborg sich bereit erklärt hat, über den Aufbau und die Entwicklung des schwedischen Frakturregisters zu berichten. In seinem Beitrag wird zum einen deutlich, welches Potenzial in diesen Registerstudien liegt, aber auch, wie groß der Aufwand sein kann, um diese in einem arztbasierten Studiensetting zu betreiben. Dies leitet über zum Beitrag von Herrn PD Dr. Beirer, der schön verdeutlicht, wie mittels patientenzentrierter Untersuchungen eine effektive und gleichzeitig valide Aussage über die Qualität unfallchirurgischer Versorgungen geleistet werden kann, die über die klassischen Score-Systeme, wie Western Ontario and McMaster Universities Osteoarthritis Index (WOMAC) oder Harris Hip Score, hinausgehen kann.

Sie sehen, dies ist ein hochspannendes Thema für die Unfallchirurgie, und daher möchte ich mich auch beim Springer Me- dizin Verlag bedanken, der es ermöglicht hat, hier dieses Leitthema zu bearbeiten.

Ich wünsche viele neue Erkenntnisse beim Lesen und stehe jederzeit gerne zum Gedankenaustausch zur Verfügung.

Ihr

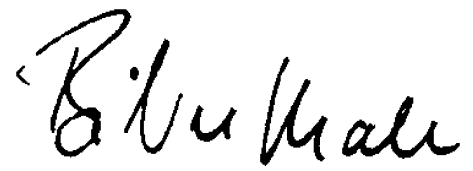

Univ.-Prof. Dr. Peter Biberthaler, München

\section{Korrespondenzadresse

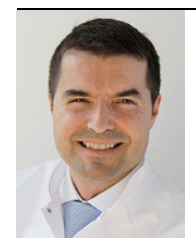 \\ Univ.-Prof. Dr. P. Biberthaler \\ Klinik und Poliklinik für Unfallchirurgie, Klinikum rechts der Isar, Technische Universität München Ismaningerstr. 22, 81675 München, Deutschland Peter.Biberthaler@mri.tum.de}

Interessenkonflikt. P. Biberthaler gibt an, dass kein Interessenkonflikt besteht.
Auch online Zugang zu allen Beiträgen Ihres Zeitschriftenabonnements

Wussten Sie, dass Sie als Abonnent dieser Zeitschrift automatisch Online-Zugriffsrechte auf das gesamte Beitragsarchiv und die CME-Kurse haben?

Der Zugang zu Ihrer Online-Bibliothek und den CME-Kursen ist im Abonnement Ihrer Zeitschrift inbegriffen. Für den Zugang müssen Sie sich lediglich einmal über www.springermedizin.de/register registrieren.

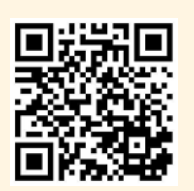

Über diesen QR-Code schnell und einfach registrieren

Bitte nutzen Sie für die Registrierung Vorund Nachname und Lieferadresse wie beim Abonnement der Zeitschrift (s. Adressaufkleber auf Ihrem Heft). So kann im System die Zugehörigkeit zu Ihrer Zeitschrift sichergestellt werden.

Aufgrund des Heilmittelwerbegesetzes dürfen die Inhalte der Website nur medizinischen Fachkreisen zur Verfügung gestellt werden. Bei der Anmeldung bitten wir Sie deshalb, einen Berufsnachweis vorzulegen.

Bei Medizinern mit Mitgliedschaft in der deutschen Ärztekammer reicht die einheitliche Fortbildungsnummer

(EFN). Alternativ schicken Sie eine Bestätigung des Arbeitgebers, Studiennachweis oder andere Zeugnisse ganz unkompliziert an kundenservice@springermedizin.de.

Mit Benutzername und Passwort haben Sie außerdem Zugang zu den freien Inhalten auf den Seiten von: www.springermedizin.de www.aerztezeitung.de

Sollten Fragen oder Probleme auftauchen, wenden Sie sich einfach an Ihren Kundenservice:

kundenservice@springermedizin.de 